\title{
A Robust Iris Localization Model Based on Phase Congruency and Least Trimmed Squares Estimation
}

\author{
Lili Pan, Mei Xie, Tao Zheng, and Jianli Ren \\ School of Electronic Engineering, University of Electronic Science and Technology of China, \\ No. 4, Section 2, Jianshe North Road, Chengdu, China \\ panlili8255@hotmail.com, xiemei@ee.uestc.edu.cn
}

\begin{abstract}
Iris localization is a crucial step in iris recognition. The previous proposed algorithms perform unsatisfactorily due to the disturbing of eyelash and variation of image brightness. To solve these problems, we proposed a robust iris position estimation algorithm based on phase congruency analysis and LTSE (Least Trimmed Squares Estimation). Through using the robust regression method to fit iris edge points we can solve the eyelash occlusion problem at a certain extent. The experimental results demonstrate the validity of this algorithm.
\end{abstract}

Keywords: Iris Segmentation, Phase Congruency, Least Trimmed Squares Estimation.

\section{Introduction}

As an important branch of biometric identification, iris recognition, which is regarded as the most secure personal identification method, attracted tremendous attention of more and more researchers and has developed rapidly over recent years[1][2][3]. A critical part influencing the performance of iris recognition is the demarcating of iris' inner and outer boundaries at pupil and sclera, for the error of this part will cause the non-iris region mapping in the normalization part. In fact, false non-match errors always arise due to the inaccuracy of localization. Therefore, a large number of iris localization algorithms having been proposed to reach the goal to localize iris boundary robustly and accurately.

The earliest iris localization algorithms, which we are most familiar with, were proposed by Daugman [4] and Wildes [5]. To reach the goal of robustness, an integrodifferential detector is adopted in [4] and Hough transform is employed in [5]. These two methods were frequently applied to the iris recognition system in the previous time; however the insufficiency in executing time and segmenting off-angle gazing iris image caused the emergence of some new methods [6-13]. These methods made progress to improve the speed and accuracy of iris segmentation a lot. Our previous attempts to iris localization are based on image intensity derivative and least squares estimation [14]. Although some progress has been made, some problems also exist. The most emergent problem needing us to solve is the tradeoff between efficiency and accuracy. 
In this paper, we propose a novel and robust iris localization algorithm based on phase congruency and least trimmed squares estimation. Phase congruency [15] [16] is a novel model for feature detection that populates features are perceived at points in an image where the Fourier components are maximally in phase. Compared with the gradient based edge detection method adopted in [4] [8-9] [14], it is not sensitive to variation in image illumination. Moreover, in this paper, we employ an improved method to compute phase congruency through automatically selecting the range of scales over which a locally one-dimensional feature exists [16]. LTS estimation [17] [18], as a robust regression method, can eliminate the influence of outliers that are produced on account of the eyelash at the time of curve fitting. It reduces the sensitivity of the fitting method [11], which is based on LSE, to fake iris edge points. Meanwhile, it improves the efficiency of the segmentation method [13] by avoiding detecting ellipse in a large region and complex iterative computation. Although, Jinyu Zuo [6] attempted to discard the worst $10 \%$ edge points to doing a fitting to increase the robustness and efficiency of the segmentation algorithm; however, this method depends heavily on the initial fitting result. The proposed algorithm of this paper solves this problem properly.

\section{Iris' Inner and Outer Edge Points Detection}

Iris' inner and outer edge point detection is the first step of iris localization and the distribution of these edge points determines the curve fitting result. Actually, for the intensity gradient based edge detection method, it is usually influenced by the variation of image illumination and contrast variation. Therefore; we select the phase congruency model for pupillary and limbic edge point detection.

\subsection{Specular Highlights Removal and Pupillary Coarse Center Detection}

As we know, most of iris images are captured under the NIR (near-infrared) illumination and the pupil area always contaminated by the reflections which are shown in Fig.1(a). These specular reflections always interrupt the pupillary edge boundary detection and reduce accuracy of segmentation. As most previous iris segmentation algorithms deal with this problem, we should firstly remove these reflection noises. In this paper, we firstly localize the reflections according to their intensity value, their location in the iris image and the sharpness of their boundaries. After we get the location of the reflection regions, we can fill these regions according to the morphological method that is proposed in [20]. One example of the reflection removal is shown in Fig.1(c).

After we remove the specular highlights, we need to find the coarse central point of the pupil to determine the region for searching pupillary and limbic edge points in the next step. The coarse central point of the pupil is an important location indication in such a dimensionless coordinate system. In order to improve the speed and accuracy of this indicating point detection, the original iris image is transformed to a binary image. The pixel, whose intensity value is below the threshold $V_{P}$ and its 8 neighborhood's intensity values are all below the threshold $V_{P}$, is set to be 0 in the binary image. Other pixels are set to be 1 . After this operation, almost only the pupil region is 
black in the binary iris image and the influence of eyelash can be reduced to the greatest extent. The threshold $V_{P}$ is:

$$
V_{p}=V_{\text {min }}+V_{\text {range }}
$$

Where $V_{\min }$ is the beginning intensity value of the iris image and $V_{\text {range }}$ is the intensity distribution range of the pupil region. It can be estimated as $k^{*} V_{\text {all }}$ (a suitable value for $k$ is set to be 0.04 through our experiments), where $V_{\text {all }}$ is the intensity distribution range of the whole iris image. Then, we adopt a hierarchical grid searching method [21] to detect the coarse center of the pupil $\left(x_{p}^{\prime}, y_{p}^{\prime}\right)$ in the binary image. Detection is carried out by scanning all possible image locations with a series circular template $\left\{S_{l}, S_{2} \ldots S_{N}\right\}$ of radius $\left\{R_{l}, R_{2} \ldots R_{N}\right\}$. At each location a matching score describes the similarity of the template and the underlying image patch. When the similarity score is lager than the threshold $T_{m}$, the underlying region is the candidate of pupil region. Through a coarse to fine manner, we can get the center location of pupil region whose similarity with the circular template is maximal. In addition, a down sampling operation to binary image is employed to increase the detection speed. In Fig. 1(c), the center and the width of the square are the coarse center of the pupil and the diameter of the matched template respectively.

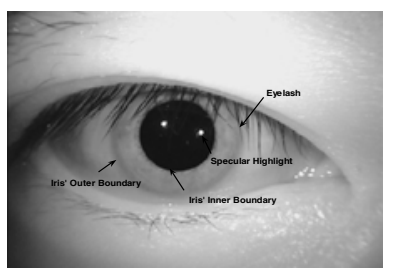

(a)

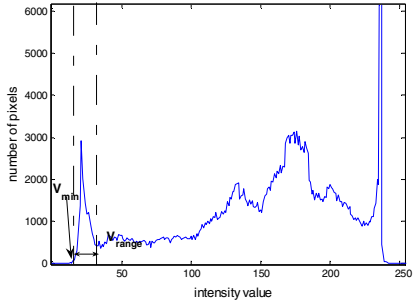

(b)

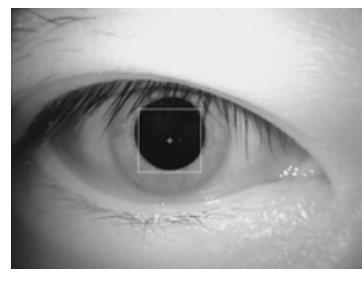

(c)

Fig. 1. (a) The original iris image that is acquired by the OKI's IRISPASS-h device. This equipment has two LED illuminators and generates two specular highlights in the pupil region of the image. (b) The intensity histogram distribution. (c) The reflection removal and the pupillary coarse center detection result.

\subsection{Edge Points Detection Based on Phase Congruency of 1-D Signal}

How to localize these edge points fast and precisely is a crucial problem. The shortage of the existing algorithms is that the edge point detection is always interrupted by illumination variation and contrast variation, and needs a comparatively long time to compute the edge map of the whole image [5] [10]. Phase congruency is an effective means for edge detection and will perform excellently when applied to iris edge point detection of course. In this work, in order to improve the speed of edge point detection, we only calculate the phase congruency of some rows in the vicinity of the pupillary coarse center and process the intensity value of a row as a 1-D signal. 
Phase congruency is a relative new model for feature detection and invariant to changes in image brightness or contrast; hence, it provides an absolute measure of the significance of feature points. Generally speaking, the peaks of phase congruency correspond to significant feature points of an image. Peter Kovesi [21] described the phase congruency model for 1-D signal as follows:

$$
P C(x)=\frac{\sum_{n} W(x)\left\lfloor A_{n}(x)\left[\cos \left(\phi_{n}(x)-\bar{\phi}(x)\right)-\left|\sin \left(\phi_{n}(x)-\bar{\phi}(x)\right)\right|-T\right]\right.}{\sum_{n} A_{n}(x)+\varepsilon}
$$

Where \lfloor\rfloor denotes that the enclosed quantity is equal to itself when its value is positive, and zero otherwise. $A_{n}$ represents the amplitude of the $n$th Fourier component of the 1-D signal, and $\phi_{n}(x)$ represents the local phase of the Fourier component at position $x$. $\varepsilon$ is a small constant to avoid division by zero. $W(x)$ is a phase congruency weighting function that is designed for sharpening the localization of features, especially those that have been smoothed and can be constructed as below:

$$
W(x)=\frac{1}{1+e^{\gamma(c-s(x))}}
$$

Where $\mathrm{c}$ is the cut-off value of filter response spread below which phase congruency values become penalized, and $\gamma$ is a gain factor that controls the sharpness of the cutoff. $s(x)$ is a measure of filter response spread. The above parameter $T$ is the total noise influence over all scales. In this work, the logarithmic Gabor function, which maintains a zero DC component, is selected to calculate the phase congruency.

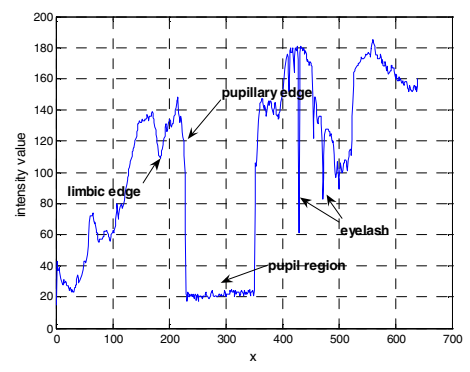

(a)

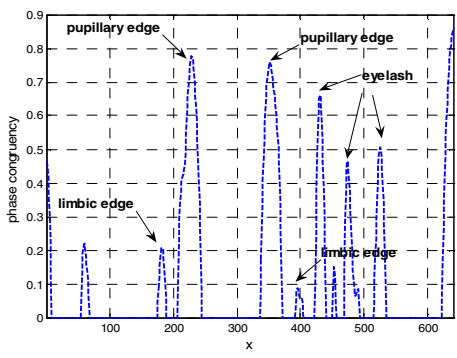

(b)

Fig. 2. (a) The intensity curve of one row near the coarse center of pupil in t Fig.1.(a). For testing the validation of the phase congruency model, we select an iris image shown in Fig.1 with relatively blurred limbic boundary to analyze. (b) The phase congruency of the left intensity curve.

In this paper, we employed the improved phase congruency algorithm proposed in [16] to localize the pupillary and limbic edge points. Compared with the limbic boundary, the pupillary boundary and eyelash is the more obvious feature in the iris image, and they give rise to peaks in phase congruency with high value which can be 
seen in Fig.2. There are two main steps to detect the pupillary edge points. First of all, we select a series of intensity values $S_{y}(x)$ of row $y$ which is near the pupillary coarse center and compute its phase congruency. An important parameter for the phase congruency calculation is the scale spread. In this paper, we estimate this parameter by tracking the phase over scale and record the start-scale and end-scale $\lambda_{b}$ and $\lambda_{e}$ at which phase is congruent on the pupillary boundary. The influence of eyelash noises in the pupil region can be eliminated through setting the noise threshold $T$ [15]. Over the selected range of scales, from $\lambda_{b}$ to $\lambda_{e}$, we compute the phase congruency $P C_{y}(x)$ of $S_{y}(x)$ and the result is shown in Fig.2. Second, we need to localize the position of these peaks that correspond to the pupillary edge points. In section 2.1, the pupillary coarse center $\left(x_{p}^{\prime}, y_{p}^{\prime}\right)$ is gained and the local maxima can be localized as below:

$$
\left\{\begin{array}{l}
\frac{\partial P C_{y}(x)}{\partial x}=0 \\
\frac{\partial^{2} P C_{y}(x)}{\partial^{2} x}<0
\end{array}\right.
$$

The pupillary edge points $x_{p l}$ and $x_{p r}$ in row $y$ are the local maximal points of phase congruency which are nearest to the pupillary inner point $\left(x_{p}^{\prime}, y_{p}^{\prime}\right)$ on the right and left respectively. Similarly, we can get a group of edge points of pupil in the rows which are near the pupillary inner point $\left(x_{p}^{\prime}, y_{p}^{\prime}\right)$.

For the contrast of limbic boundary is not so great as the pupillary boundary, limbic edge point detection is much more difficult than the pupillary edge point detection. Furthermore, it is always disturbed by the obvious fleck and stripe texture of iris and eye lash. To avoid this interruption and emphasize the intensity change in the iris' outer boundary, we need to smooth the original intensity curve $S_{y}(x)$ and set the intensity value of the pupil region in row $y$ to be the intensity value of iris region before calculating the phase congruency. The smooth operation is as below:

$$
S_{y}^{\prime}(x)=S_{y}(x) * g(x, \sigma)=\int_{-\infty}^{+\infty} S_{y}(x-u) \cdot \frac{1}{\sqrt{2 \pi} \sigma} \exp \left(-u^{2} / 2 \sigma^{2}\right) d u
$$

Where $g(x, \sigma)$ is the Gaussian smoothing function with deviation $\sigma . \sigma$ has an important effect on the selection of phase spread parameter. As we calculate the phase congruency for pupillary edge point detection, we should select a range of scales to calculate the phase congruency according to the characteristic of limbic edge. The result is shown in Fig. 3 and the limbic edge points have much higher phase congruency value. We can find that the limbic edge points of row $y$ are the local maximal points which are nearest to the pupillary inner point $\left(x_{p}^{\prime}, y_{p}^{\prime}\right)$. In the same way, we can get a group of limbic edge points through processing a number of rows which are not occluded by eyelash. We can determine whether one row is occluded by eyelash through calculate the number of peaks whose value is above 0.45 when compute the phase congruency for pupillary edge point detection because eyelashes are most obvious feature of an iris image. 


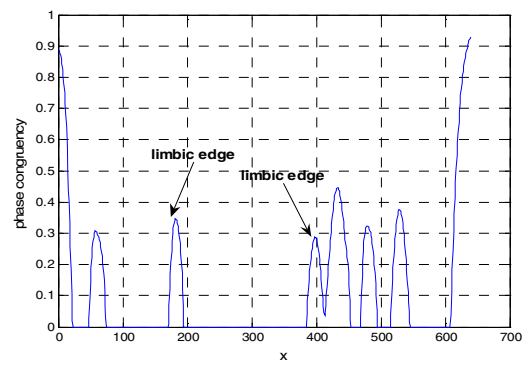

Fig. 3. The phase congruency of the smoothed intensity curve, in which the intensity values in pupil region are set to be the iris intensity value

\section{Least Trimmed Squares Estimation}

As we know, in the iris image, the boundaries of iris are almost circular contour at front-angle position. When the iris image is captured at off-angle position, the circular contour will become elliptical contour. Thus, we set two ellipses to fit iris' inner and outer boundaries. Although slight non-circular iris' boundaries exist, they won't cause any problem if we use the same approach to model the boundaries for both the enrolled and inquiry iris images. The most normal data fitting algorithm is the LSE (Least Squares Estimation) method which gives every sample the same weight. Actually, this method is notoriously sensitive to outliers, which are defined as the sample values that cause surprise in relation to the majority of the sample. In this work, the outliers are usually the no-iris edge points, such as the eyelash edge points, and even one outlier will give rise to the departure of position estimation. To increase robustness of the iris position estimation, the fitting method must be more forgiving about outlying measurements; therefore, the LTSE (Least Trimmed Squares Estimation) that is one of the robust regression methods is applied. The principle of LTSE is to minimize the sum of squares for the smallest $h$ of residuals [17] [18].

Consider an ordinary robust regression model that can be described as the followed form:

$$
Z=A \beta+v
$$

$\boldsymbol{Z}=\left[z_{1}, \ldots, z_{m}\right]^{T} \in \boldsymbol{R}^{m}$ is a measurement vector, $\boldsymbol{A}=\left[\boldsymbol{a}_{1}, \ldots, \boldsymbol{a}_{\boldsymbol{m}}\right]^{T} \in \boldsymbol{R}^{m \times n}$ is a design (or model) matrix with full column rank, $\in \boldsymbol{R}^{n}$ is an unknown parameter vector to be estimated, and $\boldsymbol{v} \in \boldsymbol{R}^{m}$ is a random noise vector. For a given vector $\in R^{n}$, the residual vector is defined as $\boldsymbol{r}() \equiv \boldsymbol{Z}-\boldsymbol{A}$, with $i$ th element $r()_{i}$. The robust least trimmed squares estimator for is given by:

$$
\min _{\beta} \sum_{i=1}^{h} r^{2}(\beta)_{i: m}
$$

Where $r^{2}()_{1: m} \leqslant \ldots \leqslant r^{2}()_{i: m}$ are the ordered squared residuals, $h$ is the trimming constant which can he set to a value in the range from $m / 2$ to $m$. Thus there will be $h$ 
data points, out of $m$, used to estimate the parameters. LTSE also employs the random sampling technique to avert the dependence on the initial fitting result [6].

The iris position estimation according to the sampled edge pionts is an elliptical fitting problem. And we know the normal equation for an ellipse is:

$$
\frac{\left(x \cos \theta+y \sin \theta-x_{o} \cos \theta-y_{o} \sin \theta\right)^{2}}{A}+\frac{\left(-x \sin \theta+y \cos \theta+x_{o} \sin \theta-y_{o} \cos \theta\right)^{2}}{B}=1
$$

Where $\left(x_{o}, y_{o}\right)$ is central location of the ellipse and $\{A, B\}$ are the major and minor axes, and $\theta$ is the orientation of the ellipse. The equivalent function can be written as below:

$$
a x^{2}+b x y+c y^{2}+d x+e y+f=0 \quad b^{2}-4 a c<0
$$

In section 2.2, we obtain a number of pupillary edge points $\left(x_{1}, y_{1}\right),\left(x_{2}, y_{2}\right), \cdots$ $\left(x_{N}, y_{N}\right)$ and the parameter $a, b, c, d, e$ can be estimated according to the followed simultaneous Equations.

$$
\left[\begin{array}{cccccc}
x_{1}^{2} & x_{1} y_{1} & y_{1}^{2} & x_{1} & y_{1} & 1 \\
x_{2}^{2} & x_{2} y_{2} & y_{2}^{2} & x_{2} & y_{2} & 1 \\
\vdots & \vdots & \vdots & \vdots & \vdots & \vdots \\
x_{N}^{2} & x_{N} y_{N} & y_{N}^{2} & x_{N} & y_{N} & 1
\end{array}\right]\left[\begin{array}{c}
a \\
b \\
c \\
d \\
e \\
f
\end{array}\right]=\left[\begin{array}{c}
0 \\
0 \\
\vdots \\
0
\end{array}\right]
$$

Here, for the pupil position estimation problem, the vector of observation $\boldsymbol{Z}$ is $[0,0, \ldots, 0]^{\mathrm{T}}$, the component of the matrix of observations on the explanatory variables $\boldsymbol{a}_{\boldsymbol{i}}$ is $\left[x_{i}^{2}, x_{i} y_{i}, y_{i}^{2}, x_{i}, y_{i}, 1\right]$, and the vector of regression coefficients to be estimated $\boldsymbol{\beta}$ is $[a, b, c, d, e, f]$. According to the direct ellipse-specific fitting method that are introduced in [19], the estimation of $\boldsymbol{\beta}$ under the LTS principle is the solution of Equ.(12) subject to $\beta^{T} \mathrm{C} \beta=1$, where $C$ is the matrix that expresses the constraint. From the above description, we can get that the weights for the outliers are 0 in the LTSE method, That is, the LTSE performs outlier rejection while fitting data. In the same way, we can get the estimation of iris position.
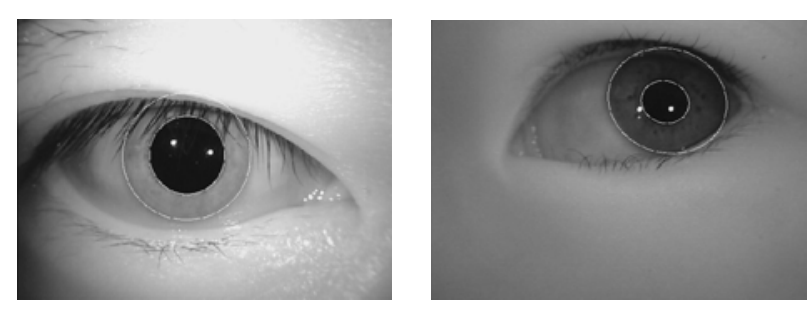

Fig. 4. The ellipse fitting results for the front-angle and off-angle captured iris image 


\section{Experiment Result}

The database used to test this proposed algorithm includes three parts. One is the public database CASIA version 2.0, and others are CASIA version 3.0(Twins) and the private database founded by ourselves. The CASIA version 2.0 database contains 1200 images with a resolution of $640 \times 480$ from 60 live human test subjects ( 20 different images of each subject) over two visitors separated by 40 days. The CASIA Version 3.0(Twins) contains 100 pairs of twins' 3183 images $(640 \times 480$ pixel resolution). The private database contains 3300 iris images $(640 \times 480$ pixel resolution) of 115 persons and the image acquisition device is OKI's IRISPASS-h.

\subsection{The Comparison of LSE and LTSE}

Though we adopt some means to eliminate the influence of eyelash occlusion, the fake pupillary and limbic edge points appear sometimes on account of the multiple eyelashes, which are overlap in a small area. In order to eliminate the effect of the fake edge points when fitting circle, we choose the LTSE method that we introduce in section 3, which can endure $50 \%$ bad points. Fig.5 gives an example of the comparison between LSE and LTSE on the iris location estimation of the same image, whose pupil and iris region is occluded by multiple eyelashes. In Fig.5(a), the scattered points are the pupillary and limbic edge points and the two groups of ellipses are the LTSE and LSE fitting result. The test on the CASIA version 2.0 database, CASIA version 3.0(Twins) database, and our private database shows that the EER reduces by $68.2 \%, 66.8 \%$ and $76.5 \%$ respectively through using LTSE rather than LSE to fit the edge points that are detected by phase congruency analysis.

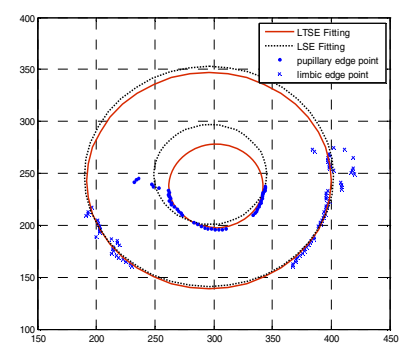

(a)

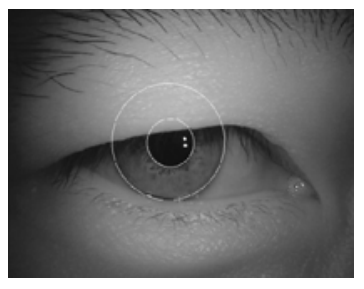

(b)

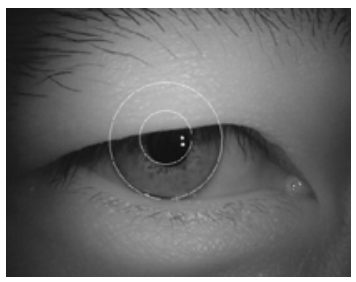

(c)

Fig. 5. The comparison of LSE and LTSE. (a) The edge point distribution and curve fitting result. (b) The iris localization result by using LTSE. (c)The iris localization result by using LSE.

\subsection{The Test Result on CASIA Database and Our Private Iris Database}

On the above database, the iris location estimation method based on phase congruency and LTSE is proved to be precise. Daugman's method [4], Wildes' method [5] and three other methods are implemented on the same database for the comparison. 
To test the performance of the proposed algorithm objectively, we adopt different methods in the iris localization part and the same feature extracting and matching methods proposed by Daugman[4] to compare the different localization algorithms' performance. We give the comparing results of these five methods in Table1. PC+LSE means first detecting the iris' inner and outer edge point through phase congruency analysis and then doing ellipse fitting to these edge points based on LSE; PC+ILSE means first detecting the iris' inner and outer edge point through phase congruency analysis and then using Improved LSE, which is proposed in [6], to fitting these edge points; PC+LTSE means first detecting the iris' inner and outer edge point through phase congruency analysis and then using LTSE to fit ellipses to these edge points. All the experiments are performed in Matlab 2006 environment on a $3.98 \mathrm{GHz}$ duo CPU (1.99G per core) and 2G DDR memory computer. Our algorithm only needs $0.13 \mathrm{~s}$ on average to localize one iris image. After we implement the proposed algorithm in $\mathrm{C}$ and optimize the code, its speed can reach $30 \mathrm{~ms} / \mathrm{f}$, that is, it can satisfy the requirement of real time iris image processing system.

Table 1. The Comparison of Iris Localization Algorithms

\begin{tabular}{|c|c|c|c|c|c|c|}
\hline & \multicolumn{2}{|c|}{$\begin{array}{c}\text { CASIA Version } 2.0 \\
\text { Database }\end{array}$} & \multicolumn{2}{|c|}{$\begin{array}{c}\text { CASIA Version 3.0- } \\
\text { Twins Database } \\
\end{array}$} & \multicolumn{2}{|c|}{ Our Private Database } \\
\hline & EER & Mean Speed & EER & Mean Speed & EER & Mean \\
\hline Daugman $^{[4]}$ & $2.12 \%$ & $0.91 \mathrm{~s}$ & $2.89 \%$ & $0.90 \mathrm{~s}$ & $1.23 \%$ & $0.92 \mathrm{~s}$ \\
\hline Wides $^{[5]}$ & $2.37 \%$ & $0.97 \mathrm{~s}$ & $2.99 \%$ & $0.98 \mathrm{~s}$ & $1.39 \%$ & $0.95 \mathrm{~s}$ \\
\hline PC+LSE & $3.75 \%$ & $0.09 \mathrm{~s}$ & $4.04 \%$ & $0.11 \mathrm{~s}$ & $2.77 \%$ & $0.10 \mathrm{~s}$ \\
\hline $\mathrm{PC}+\mathrm{ILSE}^{[6]}$ & $2.84 \%$ & $0.09 \mathrm{~s}$ & $3.68 \%$ & $0.10 \mathrm{~s}$ & $1.67 \%$ & $0.11 \mathrm{~s}$ \\
\hline PC+LTSE & $1.19 \%$ & $0.13 \mathrm{~s}$ & $1.34 \%$ & $0.14 \mathrm{~s}$ & $0.65 \%$ & $0.13 \mathrm{~s}$ \\
\hline
\end{tabular}

\section{Conclusion}

Iris recognition has a long and colorful history. So far, there are a plenty of algorithms having been proposed. In this paper, we propose a novel iris localization algorithm, which is basing on phase congruency analysis and least trimmed squares estimation. It performs robustly due to the robust regression of LTSE; moreover, it is invariant to image brightness and contrast. The experiments on three different iris databases show the validity of the proposed algorithm.

\section{Acknowledgements}

This work was supported by a grant from the National Nature Science Foundation of China (NO.60472046). 


\section{References}

1. Jain, A.K., Flynn, P., Ross, A.A.: Handbook of Biometrics. Springer, Heidelberg (2008)

2. Jain, A.K.: Biometric recognition: Q\&A. Nature 449(6), 38-40 (2007)

3. Bowyer, K.W., Hollingsworth, K., Flynn, P.J.: Image Understanding for Iris Biometrics: A Survey. Computer Vision and Image Understanding, 1-37 (2007)

4. Daugman, J.G.: High confidence visual recognition of persons by a test of statistical independence. IEEE Trans. Pattern Anal. Machine Intell. 15(11), 1148-1161 (1993)

5. Wildes, R.P.: Iris recognition: an emerging biometric technology. Proceeding of the IEEE 85(9), 1348-1363 (1997)

6. Zuo, J., Ratha, N.K., Connell, J.H.: A New Approach for Iris Segmentation. In: 2008 IEEE Computer Society Conference on Computer Vision and Pattern Recognition Workshop on Biometrics, pp. 1-6 (2008)

7. Nabti, M., Bouridane, A.: An Efficient and Fast Iris Recognition System based on a Combined Multiscale Feature Extraction technique. Pattern Recognition 41(3), 868-879 (2008)

8. Ren, X., Peng, Z., Zeng, Q., et al.: An Improved Method for Daugman's Iris Localization Algorithm. Computers in Biology and Medicine 38(1), 111-115 (2008)

9. Basit, A., Javed, M.Y.: Localization of Iris in Gray Scale Images Using Intensity Gradient. Optics and Lasers in Engineering 45(12), 1107-1114 (2007)

10. He, X., Shi, P.: A New Segmentation Approach for Iris Recognition based on Hand-held Capture Device. Pattern Recognition 40(4), 1326-1333 (2007)

11. Pundlik, S.J., Woodard, D.L., Birchfield, S.T.: Non-Ideal Iris Segmentation Using Graph Cuts. In: Pr2008 IEEE Computer Society Conference on Computer Vision and Pattern Recognition Workshop on Biometrics, pp. 23-28 (2008)

12. He, Z., Tan, T., Sun, Z., Qiu, X.: Towards Accurate and Fast Iris Segmentation for Iris Biometrics. To be appeared in IEEE Trans. Pattern Anal. Machine Intell. (2008)

13. Miyazawa, K., Ito, K., Aoki, T.: An Effective Approach for Iris Recognition Using PhaseBased Image Matching. IEEE Trans. Pattern Anal. Machine Intell. 30(10), 1741-1756 (2008)

14. Pan, L., Xie, M.: The Algorithm of Iris Image Preprocessing. In: Proceedings of the Fourth IEEE Workshop on Automatic Identification Advanced Technologies, pp. 134-138 (2005)

15. Kovesi, P.: Image features from phase congruency. Videre: A Journal of Computer Vision Research 1(3), 1-27 (1999)

16. Schenk, V.U.B., Brady, M.: Improving Phase-Congruency Based Feature Detection through Automatic Scale-Selection. In: Sanfeliu, A., Ruiz-Shulcloper, J. (eds.) CIARP 2003. LNCS, vol. 2905, pp. 121-128. Springer, Heidelberg (2003)

17. Wang, H., Suter, D.: LTSD: A Highly Efficient Symmetry-Based Robust Estimator. In: Proceeding of the Seventh International Conference on Control, Antomation, Robatlu and Vision, Singapore, pp. 332-337 (2002)

18. Bai, E.: Outliers: Inliers and the Squares Estimator in Generalized Least Trimmed System Identification. Journal of Control Theory and Application 1, 17-27 (2003)

19. Fitzgibbon, A., Pilu, M., Fisher, R.B.: Direct Least Square Fitting of Ellipse. IEEE Trans. Pattern Anal. Machine Intell. 21(5), 476-480 (1999)

20. Soille, P.: Morphological Image Analysis: Principles and Applications, pp. 173-174. Springer, Heidelberg (1999)

21. Froba, B., Emst, A., Kubleck, C.: Real-Time Face Detection Using Edge-Orientation Matching. In: The Third International Conference on Audio- and Video-Based Biometric Person Authentication, pp. 78-83 (2001) 